\title{
INCREASED ARACHIDONIC ACID-INDUCED THROMBOXANE GENERATION IMPAIRS SKELETAL MUSCLE ARTERIOLAR DILATION WITH GENETIC DYSLIPIDEMIA
}

\author{
Adam. G. Goodwill ${ }^{1,4}$, Phoebe A. Stapleton ${ }^{2,4}$, Milinda E. James ${ }^{1,4}$, Alexandre C. \\ d'Audiffret $^{3,4}$, and Jefferson C. Frisbee ${ }^{1,4}$ \\ ${ }^{1}$ Department of Physiology and Pharmacology, West Virginia University School of Medicine, \\ Morgantown, WV 26506 \\ 2 Division of Exercise Physiology, West Virginia University School of Medicine, Morgantown, WV \\ 26506 \\ ${ }^{3}$ Division of Vascular and Endovascular Surgery, West Virginia University School of Medicine, \\ Morgantown, WV 26506 \\ ${ }^{4}$ Center for Interdisciplinary Research in Cardiovascular Sciences, West Virginia University \\ School of Medicine, Morgantown, WV 26506
}

\begin{abstract}
Objective-To determine if arachidonic acid (AA)-induced skeletal muscle arteriolar dilation is altered with hypercholesterolemia in ApoE and LDLR gene deletion mice fed normal diet. This study also determined contributors to altered AA-induced dilation between dyslipidemic mice and controls; C57/B1/6J (C57).
\end{abstract}

Methods-Gracilis muscle arterioles were isolated, with mechanical responses assessed following challenge with AA under control conditions and after elements of AA metabolism pathways were inhibited. Conduit arteries from each strain were used to assess AA-induced production of $\mathrm{PGI}_{2}$ and $\mathrm{TxA}_{2}$.

Results-Arterioles from ApoE and LDLR exhibited a blunted dilation to AA versus C57. While responses were cyclooxygenase-dependent in all strains, inhibition of thromboxane synthase or blockade of $\mathrm{PGH}_{2} / \mathrm{TxA}_{2}$ receptors improved dilation in ApoE and LDLR only. AA-induced generation of $\mathrm{PGI}_{2}$ was comparable across strains, although $\mathrm{TxA}_{2}$ generation was increased in ApoE and LDLR. Arteriolar reactivity to $\mathrm{PGI}_{2}$ and $\mathrm{TxA}_{2}$ was comparable across strains.

Treatment with TEMPOL improved dilation and reduced $\mathrm{TxA}_{2}$ production with $\mathrm{AA}$ in $\mathrm{ApoE}$ and LDLR.

Conclusions-These results suggest that AA-induced arteriolar dilation is constrained in ApoE and LDLR via an increased production of TxA $\mathrm{A}_{2}$. While partially due to elevated oxidant stress, additional mechanisms contribute which are independent of acute alterations in oxidant tone.

\section{Keywords}

skeletal muscle microcirculation; endothelium-dependent dilation; vascular reactivity; mouse models of cardiovascular disease; hypercholesterolemia

Send Correspondence to: Jefferson C. Frisbee, Ph.D., Center for Interdisciplinary Research in Cardiovascular Science, Department of Physiology and Pharmacology, Robert C. Byrd Health Sciences Center, PO Box 9105, West Virginia University School of Medicine, Morgantown, WV 26505, Phone: (304) 293-6527, Fax: (304) 293-5513, jfrisbee@hsc.wvu.edu. 


\section{INTRODUCTION}

Dyslipidemia, and specifically hypercholesterolemia, has repeatedly been demonstrated to represent a strong predisposing risk factor for the development of coronary and peripheral arterial disease (1). While this increased risk for the progression of vascular disease with hypercholesterolemia is most commonly associated with an increased predisposition for the development of atherothromboses, atherosclerotic lesions and plaque depositions $(3,10,26)$, investigations into the impact of hypercholesterolemia on vascular reactivity and endothelial function, potentially as contributing mechanisms to vascular disease, is less clearly understood.

While some disparity in the prevailing literature exists (25), the general consensus is that the development of hypercholesterolemia is usually associated with a significant reduction in the bioavailability of endothelium-derived nitric oxide $(5,6,23)$, with the relatively predictable ensuing outcome of an impaired vascular reactivity in response to stimuli that are considered to have a significant contribution from this signaling molecule/pathway (i.e., flow-mediated dilation; ref. 12). In our recent study, we provided evidence suggesting that development of familial hypercholesterolemia (a genetic disorder resulting in exceptionally high low density lipoprotein [LDL] level, in the face of an otherwise relatively normal lipid profile) in the LDL receptor gene deletion mouse or type III hyperlipidemia (a condition wherein both LDL and plasma triglycerides are significantly elevated) in the apolipoprotein E gene deletion mouse, was associated with a near complete abolition of the bioavailability of endothelium-derived nitric oxide in response to imposed stimuli (22). However, this loss of vascular nitric oxide bioavailability did not result in a profound reduction in dilator reactivity, as an increased generation of dilator signaling molecules through 12/15 lipoxygenases emerged with evolution of the dyslipidemia (22), suggesting that alterations to the metabolism of arachidonic acid may be associated with hypercholesterolemia, and that these can have profound consequences for vascular function.

In 1996, the work of Pfister and colleagues $(16,17)$ strongly suggested that diet-induced hypercholesterolemia in rabbits can lead to changes in arachidonic acid metabolism, mediated via lipoxyegnase are cytochrome $\mathrm{P} 450$ epoxygenase enzymes, causing profound alterations to dilator reactivity determined in isolated aortic segments. Additionally, Srisawat et al. (21), while providing additional evidence that diet-induced hypercholesterolemia results in impaired endothelium-dependent dilation in aortic rings, determined that chronic treatment with indomethacin improved endothelial function, and was associated with reductions in urinary levels of 2,3-dinor-thromboxane $\mathrm{B}_{2}$ and 8-iso$\mathrm{PGF}_{2 \alpha}$, a stable urinary breakdown product of thromboxane $\mathrm{A}_{2}$ and a marker of chronic oxidant stress, respectively. Most recently, Pfister demonstrated that impairments to endothelium-dependent dilation in aortic rings of hypercholesterolemic rabbits were diminished in a subgroup of animals lacking a functional thromboxane receptor (15). These previous results suggest that a contributing mechanism underlying alterations to vascular reactivity under conditions of hypercholesterolemia may involve both elevated vascular oxidant stress and metabolism of arachidonic acid through cyclooxygenase pathways. However, given recent observations in our laboratory (22) and by others (25) suggesting that alterations to endothelium-dependent reactivity may reflect the specific challenge imposed rather than a global impairment, we examined alterations to dilator reactivity in response to direct challenge with arachidonic acid itself, wherein the bioavailability of endotheliumderived nitric oxide is not a significant contributing element to the net mechanical response. Using both apolipoprotein $\mathrm{E}$ and LDL receptor gene deletion mouse models of hypercholesterolemia, the hypothesis tested in the present study was that arachidonic acidinduced dilator reactivity of skeletal muscle arterioles would be impaired in the presence of profound dyslipidemia and that this would be the result of alterations to either the 
production or vascular reactivity to metabolites of arachidonic acid via cyclooxygenase, owing to the presence of an elevated oxidant stress.

\section{MATERIALS AND METHODS}

\section{Animals}

The present study used three strains of mice, the $\mathrm{C} 57 / \mathrm{Bl} / 6 \mathrm{~J}$ (C57) as the control strain and the apolipoprotein E gene deletion (B6.129P2-Apoetm1Unc/J; ApoE) and low density

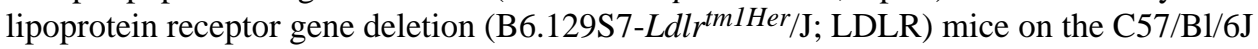
background. All mice were purchased from Jackson Laboratories (Bar Harbor, ME) at 6 weeks of age. The ApoE mouse manifests type III hyperlipidemia, in which both plasma cholesterol and triglyceride levels are elevated, although the elevations in LDL are not as severe as in the LDLR gene deletion mouse (19). In contrast, the LDLR mouse is a model of human familial hypercholesterolemia, manifesting a profound increase in serum LDL levels while ingesting a normal diet (11).

Male mice of each strain were fed standard chow and drinking water ad libitum and were housed in an AAALAC-accredited animal care facility at the West Virginia University Health Sciences Center and all protocols received prior IACUC approval. At 20 weeks of age, after an overnight fast, mice were anesthetized with injections of sodium pentobarbital $\left(50 \mathrm{mg} \cdot \mathrm{kg}^{-1}\right.$ i.p.), and received tracheal intubation to facilitate maintenance of a patent airway. In all mice, a carotid artery was cannulated for determination of arterial pressure. Blood aliquots were drawn from the jugular vein cannula for determination of glucose and insulin (Linco), a lipid profile (Waco), and nitrotyrosine (Oxis).

\section{Preparation of Isolated Skeletal Muscle Resistance Arterioles}

In anesthetized mice, the intramuscular continuation of the right gracilis artery was removed and cannulated, as described previously (8). These first order arterioles were extended to their approximate in situ length and were equilibrated at $80 \%$ of the animal's mean arterial pressure in order to approximate the in vivo intralumenal pressure experienced by the animal (13). Following equilibration, arteriolar reactivity was evaluated in response to increasing concentrations of arachidonic acid $\left(10^{-10} \mathrm{M}-10^{-6} \mathrm{M}\right.$; Sigma). Additionally, in select experiments arteriolar reactivity was also evaluated in response to increasing concentrations of prostacyclin $\left(\mathrm{PGI}_{2} ; 10^{-10} \mathrm{M}-10^{-6} \mathrm{M}\right.$; Biomol) or carbocyclic thromboxane $\mathrm{A}_{2}\left(\mathrm{TxA}_{2}\right.$; $10^{-10} \mathrm{M}-10^{-6} \mathrm{M}$; Cayman).

Removal of the arteriolar endothelium was accomplished by passing an air bolus through the perfusate line into the isolated microvessel, the efficacy of which was determined from a loss of all dilator reactivity in response to application of $10^{-6} \mathrm{M}$ acetylcholine (8). To assess the contribution of nitric oxide production or the generation of metabolites via cyclooxygenase as mediators of arteriolar reactivity, isolated vessels were treated with the nitric oxide synthase inhibitor $\mathrm{L}-\mathrm{N}^{\mathrm{G}}$-nitroarginine methyl ester (L-NAME; $10^{-4} \mathrm{M}$ for 45 minutes prior to agonist challenge; Sigma) or the cyclooxygenase antagonist indomethacin (INDO; $10^{-6} \mathrm{M}$ for 60 minutes prior to agonist challenge; Sigma), respectively. To determine the contribution of metabolites of arachidonic acid mediated via cytochrome P450 enzymes, vessels were treated with the suicide substrate inhibitor 17-octadecynoic acid (17ODYA; $10^{-5} \mathrm{M}$ for 60 minutes prior to agonist challenge; Sigma). Previous studies have demonstrated that 17-ODYA profoundly attenuates both the $\omega$-hydroxylation (producing 20hydroxyeicosatetraenoic acid; 20-HETE) and epoxygenation (producing epoxyeicosatrienoic acids; EETs) reactions of arachidonic acid through cytochrome P450 (24), thus preventing changes to vascular levels of 20-HETE or EETs as contributing mediators to endotheliumdependent dilation. To assess the contribution of lipoxygenase metabolites to the patterns of 
arteriolar dilation, vessels were treated with nordihydroguaiaretic acid (NDGA; $3 \times 10^{-5} \mathrm{M}$ for 45 minutes prior to agonist challenge; Biomol), a selective inhibitor of 12/15lipoxygenases $(20,27)$. To antagonize vascular $\mathrm{PGH}_{2} / \mathrm{TxA}_{2}$ receptors, vessels were treated with SQ-29548 ( $10^{-5} \mathrm{M}$ for 30 minutes prior to agonist challenge; Biomol), while inhibition of thromboxane synthase was accomplished using carboxyheptyl imidazole $\left(\mathrm{CHI} ; 10^{-5} \mathrm{M}\right.$ for 45 minutes prior to agonist challenge; Biomol). To reduce vascular oxidant tone, arterioles were treated with 4-Hydroxy-2,2,6,6-tetramethylpiperidine- $1-{ }^{15} \mathrm{~N}$-oxyl (TEMPOL; $10^{-4} \mathrm{M}$ for 60 minutes prior to agonist challenge, Sigma).

\section{Determination of Vascular Metabolites of Arachidonic Acid}

Vascular production of 6-keto-prostaglandin $\mathrm{F}_{1 \alpha}$ (6-keto- $\mathrm{PGF}_{1 \alpha}$; the stable breakdown product of $\mathrm{PGI}_{2}$; ref. 14), and 11-dehydro-thromboxane $\mathrm{B}_{2}$ (11-dehydro- $\mathrm{TxB}_{2}$; the stable plasma breakdown product of $\mathrm{TxA}_{2} ;$ ref. 4 ) in response to challenge with arachidonic acid within the three mouse strains was assessed using pooled conduit arteries (femoral, saphenous, iliac, carotid arteries) from each mouse. Vessels were incubated in microcentrifuge tubes in $1 \mathrm{ml}$ of physiological salt solution for 30 minutes under control conditions $\left(21 \% \mathrm{O}_{2}\right)$, after which time arachidonic acid $\left(10^{-6} \mathrm{M}\right)$ was added to the tube for an additional 30 minutes. After the second 30 minute period, the PSS was transferred to a new tube, frozen in liquid $\mathrm{N}_{2}$ and stored at $-80^{\circ} \mathrm{C}$. Metabolite release by the vessels was determined using commercially available EIA kits for 6-keto-PGF ${ }_{1 \alpha}$ and 11-dehydro-TxB 2 (Cayman).

\section{Data and Statistical Analyses}

Active tone of individual arterioles at the equilibration pressure was calculated as $(\Delta \mathrm{D} /$ $\left.D_{\max }\right) \cdot 100$, where $\Delta \mathrm{D}$ is the diameter increase from rest in response to $\mathrm{Ca}^{2+}{ }^{-}$free PSS, and $\mathrm{D}_{\max }$ is the maximum diameter measured at the equilibration pressure in $\mathrm{Ca}^{2+}$-free PSS.

Dilator responses of isolated arterioles following challenge with dilator agonists were fit with the three-parameter logistic equation:

$$
y=\min +\left[\frac{\max -\min }{1+10^{\log E D_{50}-x}}\right]
$$

where $y$ represents the change in arteriolar diameter, "min" and "max" represent the lower and upper bounds, respectively, of the change in arteriolar diameter with increasing agonist concentration, $x$ is the logarithm of the agonist concentration and $\log E D_{50}$ represents the logarithm of the agonist concentration $(x)$ at which the response $(y)$ is halfway between the lower and upper bounds.

Data are presented as mean \pm SEM. Statistically significant differences in measured and calculated parameters in the present study were determined using analysis of variance (ANOVA). In all cases, Student-Newman-Keuls post hoc test was used when appropriate and $\mathrm{p}<0.05$ was taken to reflect statistical significance.

\section{RESULTS}

Table 1 presents baseline characteristics of the mouse groups in the present study. While all mice were of similar mass at 20 weeks of age, LDLR experienced a significant elevation in mean arterial pressure and fasting insulin concentration versus values in C57 or ApoE. Additionally, both ApoE and LDLR manifested a profound hypercholesterolemia, most severe in LDLR. Further, ApoE exhibited a significant hypertriglyceridemia as well, while 
plasma triglyceride levels in LDLR were not different from that in C57. Finally, plasma levels of nitrotyrosine, a marker of chronic elevations in oxidant stress, were significantly elevated in ApoE and LDLR as compared to C57. With regard to basal vascular tone, isolated arterioles from all mouse groups demonstrated a comparable resting active diameter and passive (calcium-free) diameter, such that no significant difference in active tone was calculated between C57, ApoE and LDLR in the present study.

Data summarizing the dilator responses of skeletal muscle resistance arterioles from C57, ApoE and LDLR in response to challenge with increasing concentrations of arachidonic acid are presented in Figure 1. Under control conditions, the reactivity of arterioles from ApoE and LDLR, while not significantly different from each other, both demonstrated a reduction in their maximum bound as compared to responses in arterioles from C57. Endotheliumdenudation via perfusion with an air bolus eliminated mechanical responses of vessels across the three strains in response to application of arachidonic acid.

The effects of pharmacological blockade of lipoxygenases and cyclooxygenases with NDGA and INDO, respectively, on arachidonic acid-induced vasodilation in isolated arterioles are summarized in Figure 2. In arterioles from C57 (Panel A), blockade of lipoxygenases with NDGA had no impact on dilator responses to arachidonic acid, while treatment with indomethacin abolished all dilation to arachidonic acid. Arterioles from ApoE, while demonstrating a blunted overall reactivity to arachidonic acid, also experienced a severe reduction in dilator reactivity following cyclooxygenase inhibition with indomethacin (Panel B). However, while treatment with NDGA alone did not impact arachidonic acid-induced dilation in vessels from ApoE, application of NDGA to vessels that had been treated with indomethacin eliminated the residual dilation in response to arachidonic acid that remained following cyclooxygenase inhibition alone. Finally, arterioles from LDLR appeared to demonstrate a dilator response to arachidonic acid challenge that was dependent on the production of metabolites generated via both lipoxygenases and cyclooxygenases, as antagonists to these pathways given in isolation resulted in modest reductions to the compromised level of reactivity, while treatment with both NDGA and indomethacin abolished all arachidonic acid-induced reactivity (Panel C). Treatment of isolated arterioles from C57, ApoE or LDLR with either L-NAME or 17-ODYA did not result in either significant or consistent effects of dilator responses following challenge with increasing concentrations of arachidonic acid (data not shown).

Figure 3 presents the effects of antagonizing thromboxane $\mathrm{A}_{2}$ generation (with $\mathrm{CHI}$ ) and action (with SQ-29548) on dilator responses of skeletal muscle arterioles in the present study. In control animals, application of either CHI or SQ-29548 had no impact on arteriolar dilation in response to increasing concentrations of arachidonic acid (Panel A). In contrast, arterioles from both ApoE (Panel B) and LDLR (Panel C) exhibited a significant improvement to their degree of arachidonic acid-induced dilation relative to untreated conditions following either inhibition of thromboxane synthase with $\mathrm{CHI}$ or blockade of the $\mathrm{PGH}_{2} / \mathrm{TxA}_{2}$ receptor (SQ-29548).

Data describing the arachidonic acid-induced generation of the cyclooxygenase products $\mathrm{PGI}_{2}$ (estimated from levels of 6-keto-PGF $1 \alpha$ ) and $\mathrm{TxA}_{2}$ (estimated from levels of 11dehydro $\mathrm{TxB}_{2}$ ) from pooled arteries of the three mouse groups in the present study are summarized in Figure 4. Following application of $10^{-6} \mathrm{M}$ arachidonic acid, arteries from C57, ApoE and LDLR all demonstrated a significant increase in $\mathrm{PGI}_{2}$ release, the degree of which was comparable between the three mouse strains (Panel A). In contrast, arachidonic acid-induced generation of $\mathrm{TxA}_{2}$, while statistically significant in arteries from C57, demonstrated a substantially more robust response in vessels from both ApoE and LDLR 
(Panel B). Pre-treatment of pooled vessels with either CHI or indomethacin severely attenuated all arachidonic acid-induced $\mathrm{TxA}_{2}$ generation in all three strains.

Arteriolar reactivity in response to challenge with prostacyclin (Panel A) or carbocyclic thromboxane $\mathrm{A}_{2}$ (Panel $\mathrm{B}$ ) in the three mouse groups is summarized in Figure 5. In response to increasing concentrations of prostacyclin, arterioles from C57 and ApoE demonstrated a very similar degree of dilator reactivity, although this response demonstrated a trend toward impairment in vessels from LDLR as compared to that in vessels from either other strain (Panel A). Arterioles from all three mouse strains exhibited very similar patterns of constrictor reactivity in response to challenge with increasing concentrations of carbocyclic thromboxane $\mathrm{A}_{2}$ (Panel B).

Figure 6 presents the effects of treating vessels with the antioxidant TEMPOL, the thromboxane synthase inhibitor $\mathrm{CHI}$, or both, on arteriolar responses to increasing concentrations of arachidonic acid. Addition of TEMPOL did not have a significant impact on arteriolar diameter in vessels from any of the three mouse strains under resting conditions. In arterioles from C57 (Panel A), neither treatment with TEMPOL nor CHI had a significant impact on dilator reactivity to arachidonic acid. In contrast, for arterioles from both ApoE (Panel B) and LDLR (Panel C), treatment with either TEMPOL or CHI significantly improved dilator responses to arachidonic acid, with the effects of $\mathrm{CHI}$ being stronger than that for TEMPOL. Interestingly, in both ApoE and LDLR, combined treatment with CHI and TEMPOL did not have any effect on arachidonic acid-induced dilation beyond that determined for $\mathrm{CHI}$ treatment alone.

Figure 7 presents data describing the effects of treating arteries from C57, ApoE or LDLR with TEMPOL on arachidonic acid-induced thromboxane $A_{2}$ production. While treatment with the antioxidant had an insignificant impact on vascular thromboxane production in C57, incubation of vessels with TEMPOL significantly reduced the arachidonic acidinduced production of $\mathrm{TxA}_{2}$ in both ApoE and LDLR. However, this reduction in thromboxane generation was only partial in nature, and levels of TxA $\mathrm{A}_{2}$ production in response to challenge with arachidonic acid following treatment with TEMPOL remained significantly increased versus that in untreated arteries from ApoE and LDLR.

\section{DISCUSSION}

Although hypercholesterolemia represents a powerful risk factor for the development of peripheral artery disease (1), the effects of hypercholesterolemia on vascular reactivity and endothelial function is less clearly understood. Given recent studies suggesting that dietinduced hypercholesterolemia can alter arachidonic acid metabolism and profoundly impact vascular reactivity through signaling mechanisms associated with the generation of thromboxane $A_{2}(15,18,21)$, the present study determined the effects of genetic hypercholesterolemia on the dilator reactivity of skeletal muscle resistance arterioles in response to challenge with arachidonic acid. More specifically, the hypothesis tested in this study was that arachidonic acid-induced arteriolar dilation in ApoE and LDLR would be impaired owing to either the production of, or vascular reactivity to, metabolites of arachidonic acid via cyclooxygenase, and that these alterations would be associated with an elevated oxidant stress.

Contrary to our results with dilator stimuli that are more strongly dependent on the bioavailability of endothelium-derived nitric oxide, where reactivity was largely maintained in the face of a profound reduction in this parameter (22), the results presented in Figure 1 indicate that skeletal muscle arteriolar dilation in response to increasing concentrations of arachidonic acid was significantly reduced in both ApoE and LDLR as compared to 
responses determined in C57. Interestingly, the data presented in this figure also strongly suggest that not only is the overwhelming majority of dilator reactivity in response to arachidonic acid dependent on a functional endothelium in control animals, the impairments to arteriolar dilation with this stimulus may also originate with alterations to endothelial function, rather than within vascular smooth muscle.

While results from the present study did not demonstrate a role for either nitric oxide bioavailability or for metabolites of arachidonic acid mediated via cytochrome P450 enzymes in terms of contributing to the arachidonic acid-induced dilator reactivity of skeletal muscle arterioles in any of the three mouse strains, activity mediated through cyclooxygenase (and to a lesser extent lipoxygenase) were critical. While arteriolar dilation in response to arachidonic acid was mediated entirely via cyclooxygenase in vessels from C57, vessels from ApoE and LDLR demonstrated a dilator response that was increasingly a function of metabolites via both cyclooxygenase and lipoxygenase, with this effect being more pronounced in LDLR than in ApoE, where the response was still predominantly cyclooxygenase-dependent. However, the data presented in Figure 2 do not provide significant insight into the impaired dilator reactivity demonstrated in arterioles of $\mathrm{ApoE}$ and LDLR in response to challenge with arachidonic acid beyond the critical involvement of cyclooxygenase. Given previous studies suggesting that the development of the hypercholesterolemic condition can profoundly impact arachidonic acid metabolism in general $(7,18)$, and the recent studies from both Pfister (15) and Srisawat et al. (21) that implicate altered behavior mediated through thromboxane generation/action as contributing mechanism to altered patterns of vascular reactivity with hypercholesterolemia, we treated vessels from ApoE and LDLR with an inhibitor of thromboxane synthase (CHI) or an antagonist for the $\mathrm{PGH}_{2} / \mathrm{TxA}_{2}$ receptor (SQ-29548). As shown in Figure 3, while neither of these agents had a significant role in the dilator responses in arterioles from C57, application of either CHI or SQ-29548 resulted in a significant improvement in the dilator responses of arterioles from ApoE or LDLR in response to challenge with increasing concentrations of arachidonic acid. Interestingly, the ameliorative effect was comparable with either pharmacological agent. While this implicates either increased thromboxane generation or an increased gain/sensitivity at the vascular thromboxane receptor as contributing mechanisms to the impaired arachidonic acid-induced arteriolar dilation, these data do not provide insight into which component may be most responsible. However, these data do strongly suggest that the development of a thromboxane-sensitive component which may act to constrain arachidonic acid-induced arteriolar dilation accompanies the evolution of genetic hypercholesterolemia.

As both CHI and SQ-29548 elicited similar improvements to arteriolar dilation in response to arachidonic acid challenge in ApoE and LDLR, it was necessary to discern which processes contributed to the constrained dilator reactivity: 1) increasing thromboxane $\mathrm{A}_{2}$ production in response to arachidonic acid production, 2) increased vascular reactivity to produced thromboxane $\mathrm{A}_{2}$, or both. The data presented in Figure 4 indicate that arachidonic acid-induced generation of $\mathrm{PGI}_{2}$ (estimated from 6-keto-PGF ${ }_{1 \alpha}$ levels) remained intact in arteries of ApoE and LDLR as compared to that determined in C57, an observation that is consistent with previous studies in the coronary vasculature of ApoE mice (9). In contrast, arachidonic-acid induced generation of thromboxane $\mathrm{A}_{2}$ (estimated from 11-dehydro- $\mathrm{TxB}_{2}$ levels) was significantly increased with the evolution of genetic hypercholesterolemia in ApoE and LDLR. When taken together with data in Figure 5, which suggest that the sensitivity of resistance arterioles from ApoE and LDLR in response to increasing concentrations of either prostacyclin or thromboxane $\mathrm{A}_{2}$ is not dramatically altered from that determined for $\mathrm{C} 57$ control mice, these data may provide compelling evidence that a predominant contributing mechanism underlying the constrained arteriolar dilation with increasing concentrations of arachidonic acid may be the development of an increased 
generation of the constrictor prostanoid thromboxane $\mathrm{A}_{2}$, which antagonizes the dilator effects associated with the generation of prostacyclin.

Given that previous studies have clearly demonstrated the critical role for elevated oxidant tone in the increased generation of thromboxane through cyclooxygenase in response to challenge with arachidonic acid $(2,28,29)$, and our observations of an increase in the plasma levels of nitrotyrosine in the ApoE and LDLR as compared to that determined in C57, the data presented in Figures 6 and 7 provide some insight into the potential role that elevated vascular oxidant tone may play in the increased arachidonic acid-induced thromboxane $\mathrm{A}_{2}$ generation with genetic dyslipidemia. While treatment with TEMPOL had no impact on arachidonic acid-induced dilation or $\mathrm{TxA}_{2}$ generation in arterioles from C57, it significantly improved the dilator response in microvessels from both ApoE and LDLR and reduced the levels of $\mathrm{TxA}_{2}$ production. However, in vessels from both strains, this improvement in dilator reactivity following treatment with the antioxidant was less pronounced than that determined following treatment with the inhibitor of thromboxane synthase, CHI. Further, combined treatment with both TEMPOL and CHI did not result in an improvement beyond that determined with CHI treatment alone. Additionally, while pre-treatment of pooled vessels with TEMPOL lowered arterial thromboxane production in response to challenge with arachidonic acid, the levels of thromboxane production remained significantly elevated despite the addition of the antioxidant. Taken together these results suggest that, while an enhanced arachidonic acid-induced genesis of thromboxane $\mathrm{A}_{2}$ via thromboxane synthase represents a strong contributor to the constrained dilator reactivity in skeletal muscle arterioles of ApoE and LDLR mice, the presence of an elevated vascular oxidant tone may represent a partial contributor to this shift in the metabolism of arachidonic acid. Clearly, these results suggest that other parameters, independent of acute changes in vascular oxidant tone, also contribute to this increased generation of thromboxane $A_{2}$. Potential avenues for ongoing investigation in this regard can include the study of not only the effects of chronic elevations in vascular oxidant tone, but also the progression of a chronic state of inflammation associated with dyslipidemia $(10,26)$ and how these processes can ultimately impact pathways of arachidonic acid metabolism.

In summary, with the development of genetic hypercholesterolemia in ApoE and LDLR mice, the dilator reactivity of skeletal muscle resistance arterioles in response to increasing concentrations of arachidonic acid is impaired. This impairment does not appear to be associated with a reduction in the generation/release of, or an altered arteriolar reactivity to, prostacyclin. However, with the evolution of this dyslipidemic condition, there appears to be an increase in the arachidonic acid-induced generation of the vasoconstrictor metabolite thromboxane $\mathrm{A}_{2}$. While there does not appear to be an alteration to the arteriolar constrictor reactivity to thromboxane, the increased generation of this metabolite may compete with the dilator effects of prostacyclin, thus limiting net dilator reactivity in response to arachidonic acid. Further, while an increase in vascular oxidant stress appears to contribute to this response, additional mechanisms which are independent of acute alterations to oxidant tone also contribute to this effect. Future investigation will be required to discern which mechanistic alterations associated with the development of hypercholesterolemia contribute to the increased production of thromboxane $\mathrm{A}_{2}$, and what the implications of this shift in the metabolism of arachidonic acid are for issues such as the integrated control of tissue perfusion, tissue oxygenation and the protection from atherogenesis and atherothrombosis.

\section{Acknowledgments}

The authors gratefully acknowledge the support provided through the Translational Research Core in the Center for Interdisciplinary Research in Cardiovascular Sciences at the West Virginia University Health Sciences Center in the performance of this study. 
Support: This study was supported by the American Heart Association (EIA 0740129N) and the National Institutes of Health (R01 DK64668).

\section{References}

1. American Heart Association Statistical Summary Sheets. High Blood Cholesterol and Other Lipids. 2007. http://www.americanheart.org/presenter.jhtml?identifier=3000945

2. Bachschmid M, Thurau S, Zou MH, Ullrich V. Endothelial cell activation by endotoxin involves superoxide/NO-mediated nitration of prostacyclin synthase and thromboxane receptor stimulation. FASEB J. 2003; 17:914-6. [PubMed: 12670882]

3. Bhatt DL, Steg PG, Ohman EM, Hirsch AT, Ikeda Y, Mas JL, Goto S, Liau CS, Richard AJ, Rother J, Wilson PW. REACH Registry Investigators. International prevalence, recognition, and treatment of cardiovascular risk factors in outpatients with atherothrombosis. JAMA. 2006; 295:180-9. [PubMed: 16403930]

4. Catella F, Healy D, Lawson JA, FitzGerald GA. 11-Dehydrothromboxane $\mathrm{B}_{2}$ : a quantitative index of thromboxane $\mathrm{A}_{2}$ formation in the human circulation. Proc Natl Acad Sci U S A. 1986; 83:58615. [PubMed: 3461463]

5. de Jongh S, Lilien MR, op't Roodt J, Stroes ES, Bakker HD, Kastelein JJ. Early statin therapy restores endothelial function in children with familial hypercholesterolemia. J Am Coll Cardiol. 2002; 40:2117-21. [PubMed: 12505222]

6. Engler MM, Engler MB, Malloy MJ, Chiu EY, Schloetter MC, Paul SM, Stuehlinger M, Lin KY, Cooke JP, Morrow JD, Ridker PM, Rifai N, Miller E, Witztum JL, Mietus-Snyder M. Antioxidant vitamins $\mathrm{C}$ and $\mathrm{E}$ improve endothelial function in children with hyperlipidemia: Endothelial Assessment of Risk from Lipids in Youth (EARLY) Trial. Circulation. 2003; 108:1059-63. [PubMed: 12912807]

7. Feletou M, Vanhoutte PM. Endothelial dysfunction: a multifaceted disorder (The Wiggers Award Lecture). Am J Physiol Heart Circ Physiol. 2006; 291:H985-1002. [PubMed: 16632549]

8. Frisbee JC, Maier KG, Falck JR, Roman RJ, Lombard JH. Integration of hypoxic dilation signaling pathways for skeletal muscle resistance arteries. Am J Physiol Regul Integr Comp Physiol. 2002; 283:R309-19. [PubMed: 12121842]

9. Godecke A, Ziegler M, Ding Z, Schrader J. Endothelial dysfunction of coronary resistance vessels in apoE-/- mice involves NO but not prostacyclin-dependent mechanisms. Cardiovasc Res. 2002; 53:253-62. [PubMed: 11744035]

10. Hansson GK. Inflammation, atherosclerosis, and coronary artery disease. N Engl J Med. 2005; 352:1685-95. [PubMed: 15843671]

11. Ishibashi S, Brown MS, Goldstein JL, Gerard RD, Hammer RE, Herz J. Hypercholesterolemia in low density lipoprotein receptor knockout mice and its reversal by adenovirus-mediated gene delivery. J Clin Invest. 1993; 92:883-93. [PubMed: 8349823]

12. Joyner MJ, Dietz NM. Nitric oxide and vasodilation in human limbs. J Appl Physiol. 1997; 83:1785-96. [PubMed: 9390947]

13. Liu Y, Fredricks KT, Roman RJ, Lombard JH. Response of resistance arteries to reduced PO2 and vasodilators during hypertension and elevated salt intake. Am J Physiol. 1997; 273:H869-77. [PubMed: 9277505]

14. Nies AS. Prostaglandins and the control of the circulation. Clin Pharmacol Ther. 1986; 39:481-8. [PubMed: 3084155]

15. Pfister SL. Aortic thromboxane receptor deficiency alters vascular reactivity in cholesterol-fed rabbits. Atherosclerosis. 2006; 189:358-63. [PubMed: 16515789]

16. Pfister SL, Campbell WB. Contribution of arachidonic acid metabolites to reduced norepinephrineinduced contractions in hypercholesterolemic rabbit aortas. J Cardiovasc Pharmacol. 1996; 28:784-91. [PubMed: 8961076]

17. Pfister SL, Spitzbarth N, Edgemond W, Campbell WB. Vasorelaxation by an endothelium-derived metabolite of arachidonic acid. Am J Physiol. 1996; 270:H1021-30. [PubMed: 8780199]

18. Pfister SL, Falck JR, Campbell WB. Enhanced synthesis of epoxyeicosatrienoic acids by cholesterol-fed rabbit aorta. Am J Physiol. 1991; 261:H843-52. [PubMed: 1887929] 
19. Piedrahita JA, Zhang SH, Hagaman JR, Oliver PM, Maeda N. Generation of mice carrying a mutant apolipoprotein $\mathrm{E}$ gene inactivated by gene targeting in embryonic stem cells. Proc Natl Acad Sci U S A. 1992; 89:4471-5. [PubMed: 1584779]

20. Salari H, Braquet P, Borgeat P. Comparative effects of indomethacin, acetylenic acids, 15-HETE, nordihydroguaiaretic acid and BW755C on the metabolism of arachidonic acid in human leukocytes and platelets. Prostaglandins Leukot Med. 1984; 13:53-60. [PubMed: 6424136]

21. Srisawat S, Phivthong-Ngam L, Unchern S, Chantharaksri U, Govitrapong P, Sanvarinda Y. Improvement of vascular function by chronic administration of a cyclo-oxygenase inhibitor in cholesterol-fed rabbits. Clin Exp Pharmacol Physiol. 2003; 30:405-12. [PubMed: 12859434]

22. Stapleton PA, Goodwill AG, James ME, Frisbee JC. Altered mechanisms of endotheliumdependent dilation in skeletal muscle arterioles with genetic hypercholesterolemia. Am J Physiol Regul Integr Comp Physiol. 2007; 293:R1110-R1119. [PubMed: 17626122]

23. Stokes KY, Cooper D, Tailor A, Granger DN. Hypercholesterolemia promotes inflammation and microvascular dysfunction: role of nitric oxide and superoxide. Free Radic Biol Med. 2002; 33:1026-36. [PubMed: 12374614]

24. Wang MH, Brand-Schieber E, Zand BA, Nguyen X, Falck JR, Balu N, Schwartzman ML. Cytochrome P450-derived arachidonic acid metabolism in the rat kidney: characterization of selective inhibitors. J Pharmacol Exp Ther. 1998; 284:966-73. [PubMed: 9495856]

25. Wolfle SE, de Wit C. Intact endothelium-dependent dilation and conducted responses in resistance vessels of hypercholesterolemic mice in vivo. J Vasc Res. 2005; 42:475-82. [PubMed: 16155363]

26. Zadelaar S, Kleemann R, Verschuren L, de Vries-Van der Weij J, van der Hoorn J, Princen HM, Kooistra T. Mouse models for atherosclerosis and pharmaceutical modifiers. Arterioscler Thromb Vasc Biol. 2007; 27:1706-21. [PubMed: 17541027]

27. Zhang DX, Gauthier KM, Chawengsub Y, Holmes BB, Campbell WB. Cyclooxygenase- and lipoxygenase-dependent relaxation to arachidonic acid in rabbit small mesenteric arteries. Am J Physiol Heart Circ Physiol. 2005; 288:H302-9. [PubMed: 15388505]

28. Zou MH, Cohen R, Ullrich V. Peroxynitrite and vascular endothelial dysfunction in diabetes mellitus. Endothelium. 2004; 11:89-97. [PubMed: 15370068]

29. Zou MH, Leist M, Ullrich V. Selective nitration of prostacyclin synthase and defective vasorelaxation in atherosclerotic bovine coronary arteries. Am J Pathol. 1999; 154:1359-65. [PubMed: 10329589] 


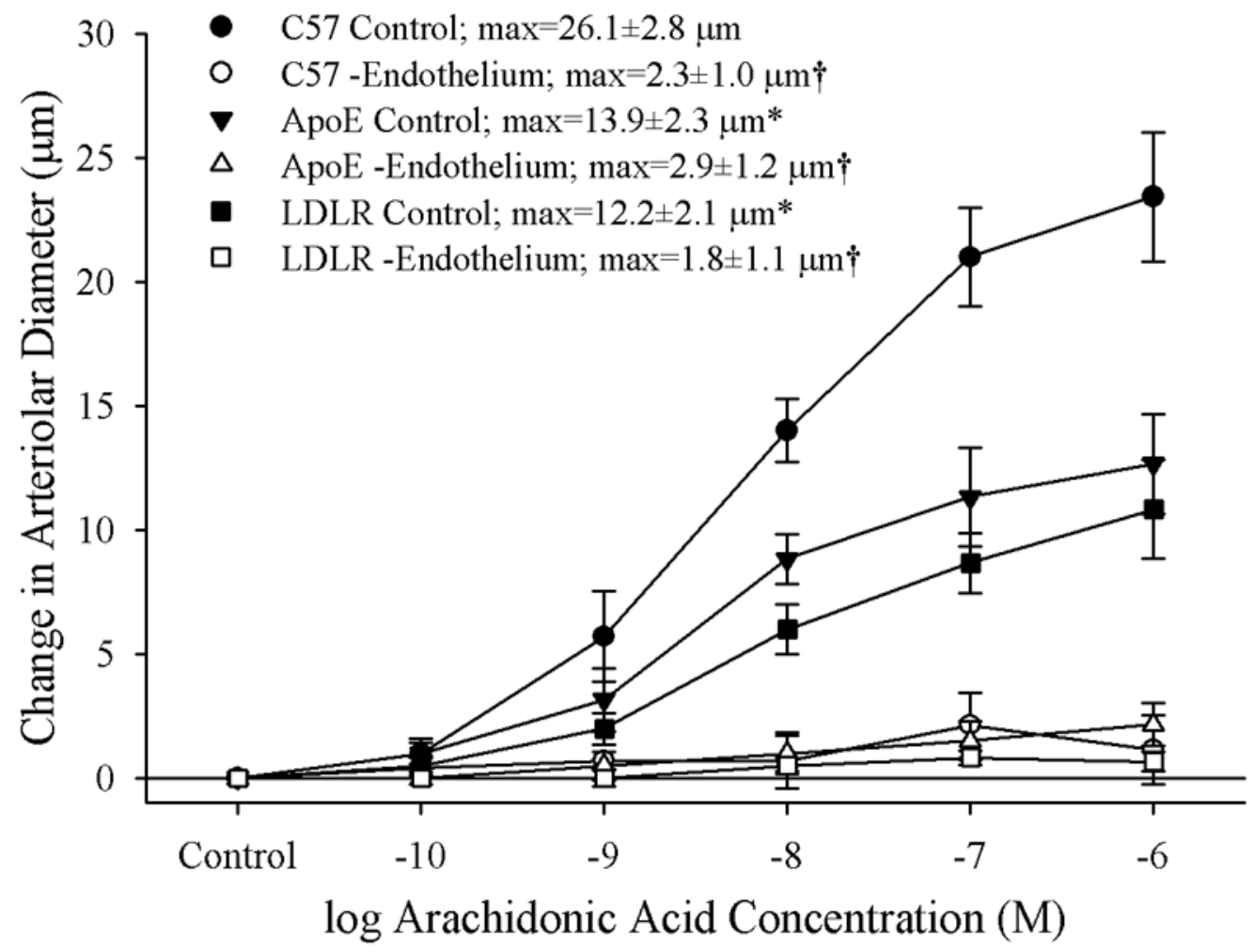

Figure 1.

Data describing the dilator reactivity of isolated skeletal muscle resistance arterioles of C57, ApoE and LDLR mice in response to increasing concentrations of arachidonic acid. Data, presented as mean \pm SEM, are shown for arterioles under control conditions and following removal of the vascular endothelium using air bolus perfusion (please see text for details). $\mathrm{n}=6$ animals for each strain; $* \mathrm{p}<0.05$ vs. $\mathrm{C} 57 ; \dagger \mathrm{p}<0.05$ vs. control within that strain. 

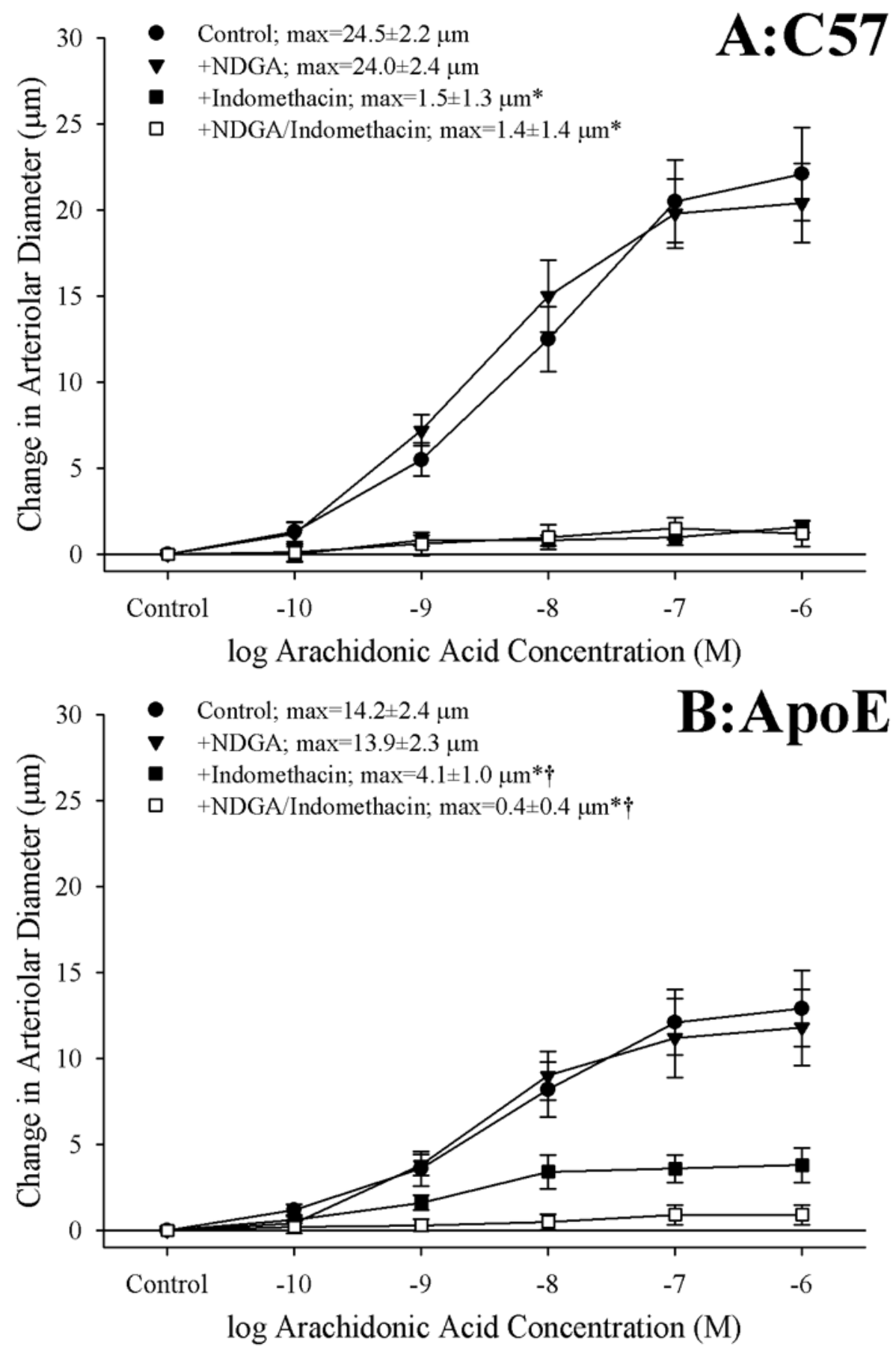


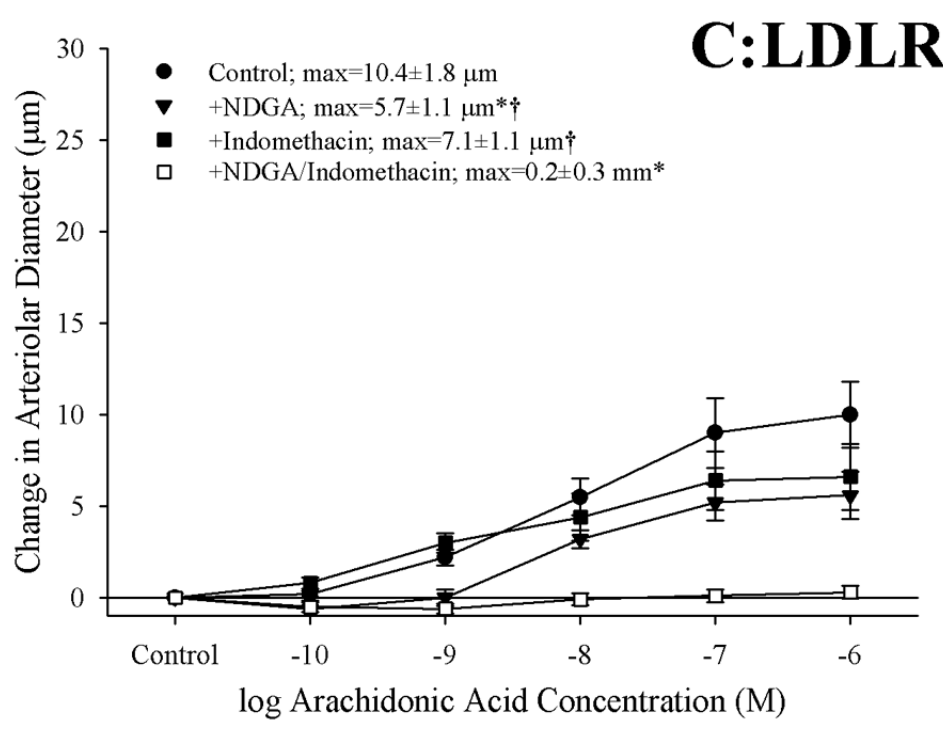

Figure 2.

Data describing the dilator responses of isolated skeletal muscle resistance arterioles of C57 (Panel A), ApoE (Panel B) and LDLR (Panel C) mice in response to increasing concentrations of arachidonic acid. Data, presented as mean \pm SEM, are shown for arterioles under control conditions, and following pharmacological inhibition of cyclooxygenases with indomethacin, lipoxygenases with NDGA or combined inhibition of both enzymatic pathways (please see text for details). $\mathrm{n}=5-10$ animals for each group; * $\mathrm{p}<0.05$ vs. control conditions, $\dagger \mathrm{p}<0.05$ vs. no response. 

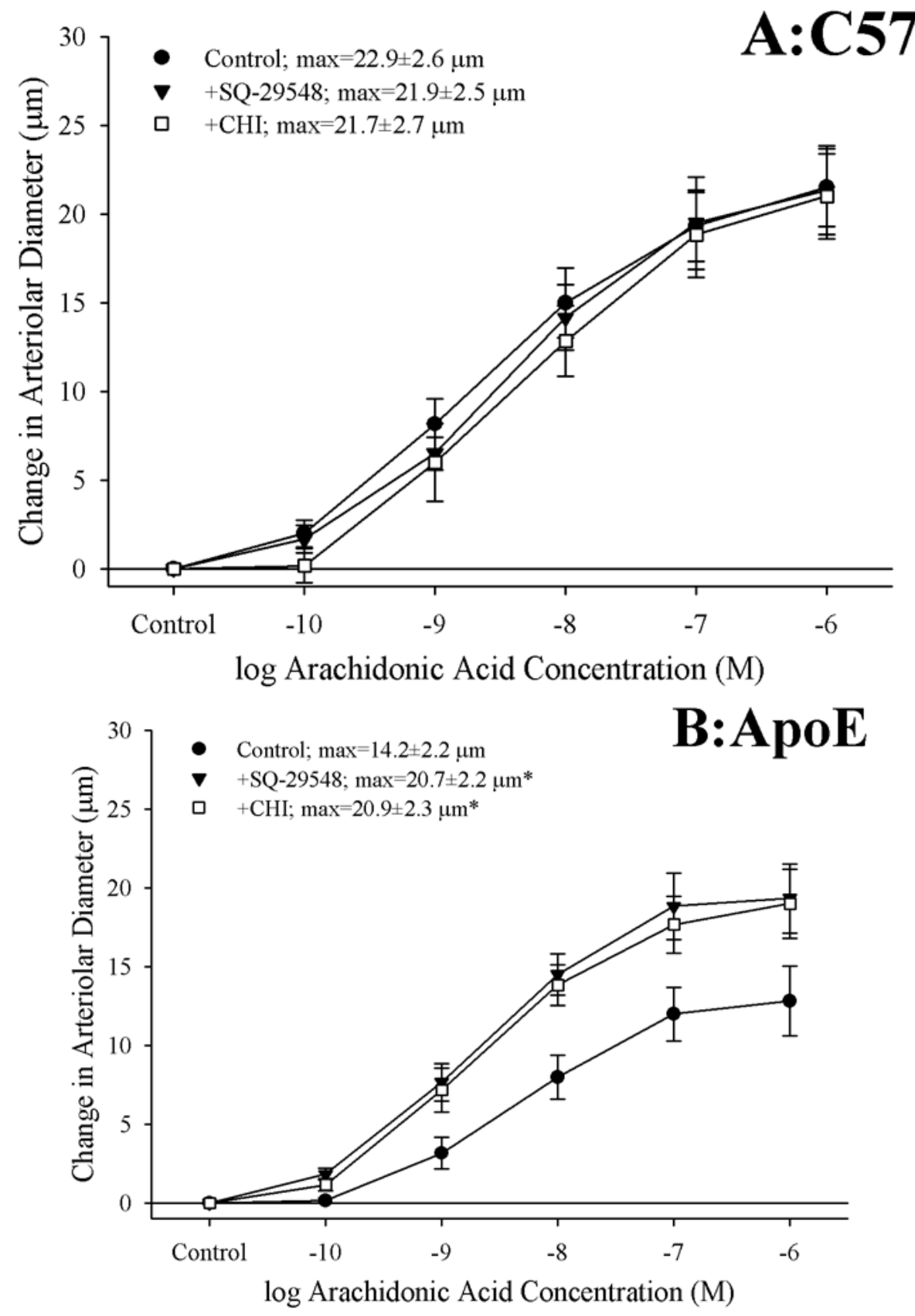


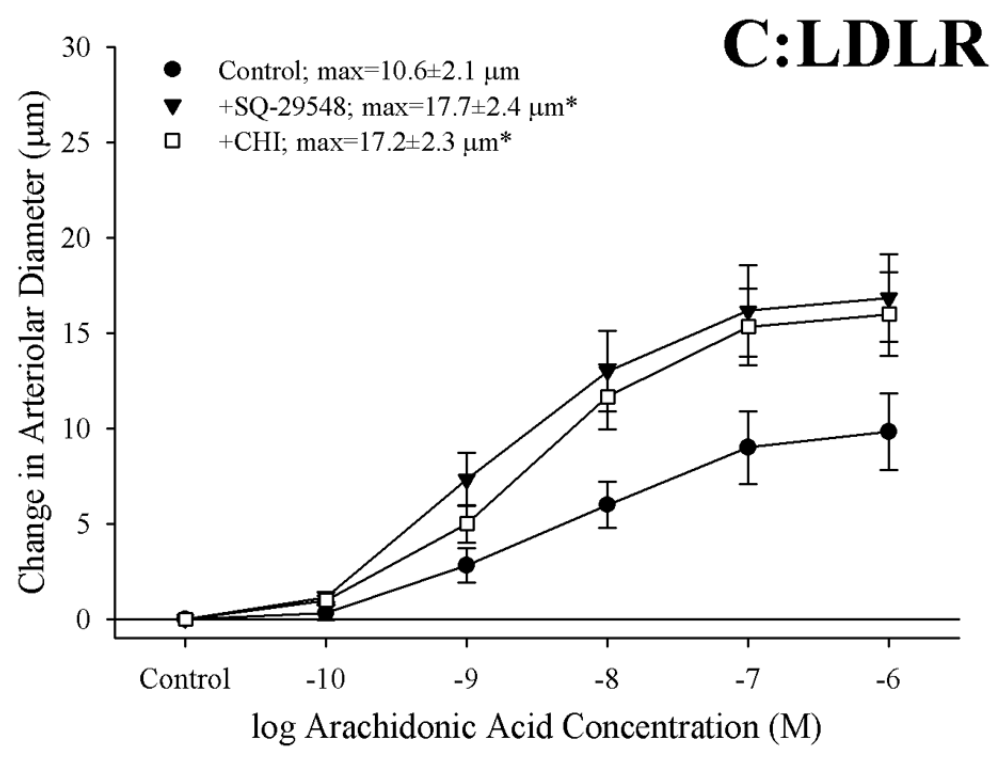

Figure 3.

Data describing the dilator responses of isolated skeletal muscle resistance arterioles of C57 (Panel A), ApoE (Panel B) and LDLR (Panel C) mice in response to increasing concentrations of arachidonic acid. Data, presented as mean \pm SEM, are shown for arterioles under control conditions, and following pharmacological inhibition of $\mathrm{PGH}_{2} / \mathrm{TxA}_{2}$ receptors with SQ-29548 and thromboxane synthase with CHI (please see text for details). $n=6-7$ animals for each group; * $\mathrm{p}<0.05$ vs. control conditions. 

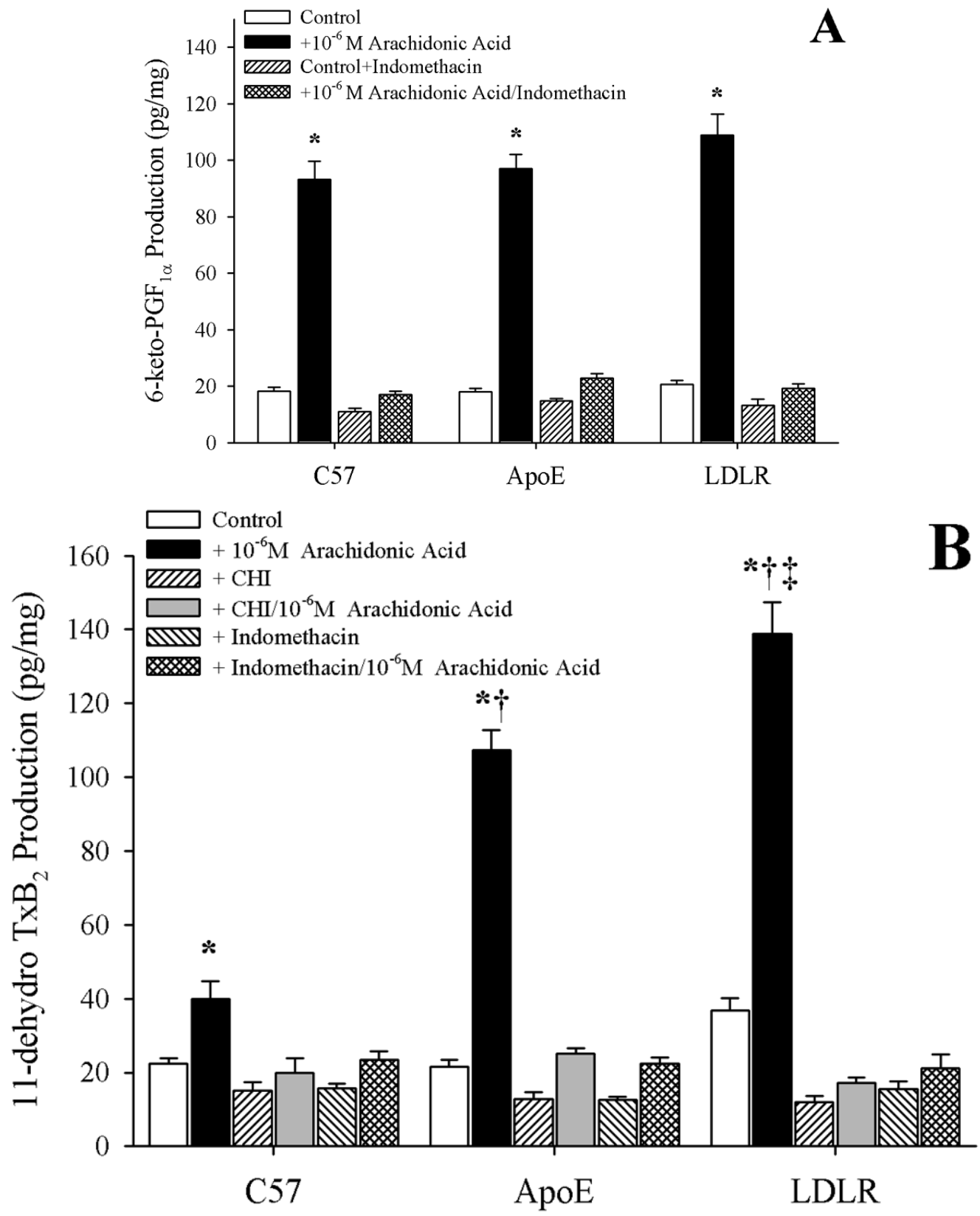

Figure 4.

Data describing the arterial production of prostacyclin (as 6-keto-PGF $1 \alpha$; Panel A) or thromboxane $\mathrm{A}_{2}$ (as 11-dehydro $\mathrm{TxB}_{2}$; Panel B) from C57, ApoE and LDLR in response to $10^{-6} \mathrm{M}$ arachidonic acid. Data, presented as mean $\pm \mathrm{SEM}$, are shown under control conditions, and following pharmacological inhibition of cyclooxygenase with indomethacin or thromboxane synthase (with $\mathrm{CHI}$ ), as appropriate. $\mathrm{n}=8$ animals for each group, with each $\mathrm{n}$ representing pooled arteries from an individual mouse; please see text for details. ${ }^{*} \mathrm{p}<0.05$ vs. respective control; $\uparrow \mathrm{p}<0.05$ vs. $\mathrm{C} 57$ under that condition; $\ddagger$ vs. ApoE under that condition. 

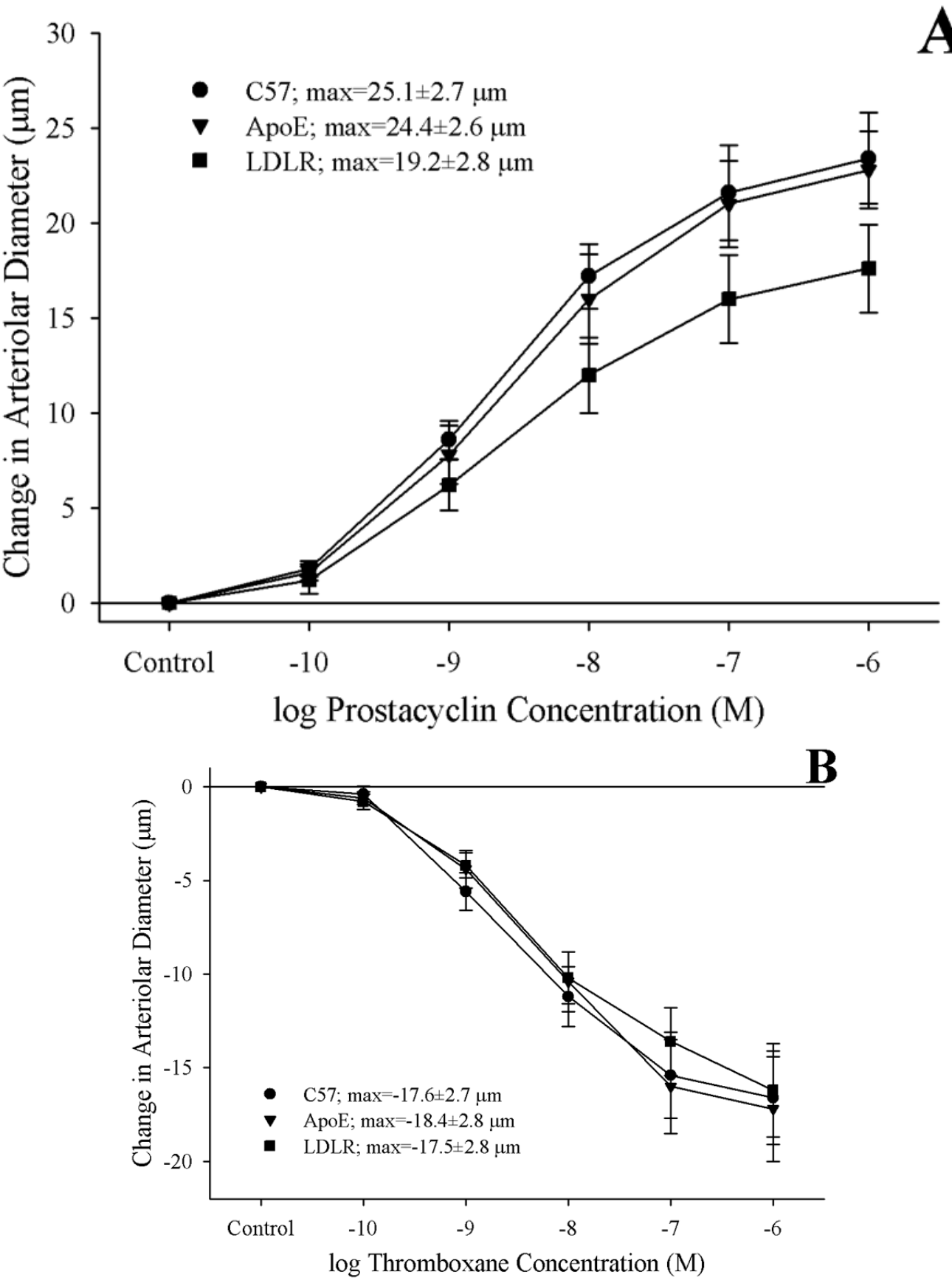

Figure 5.

Data (mean \pm SEM) describing the reactivity of isolated skeletal muscle resistance arterioles of C57, ApoE and LDLR mice in response to increasing concentrations of prostacyclin (Panel A) or carbocyclic thromboxane $A_{2}$ (Panel B). $n=6$ animals for each group, no significant differences were identified in the vascular reactivity in response to increasing concentrations of prostacyclin or thromboxane $\mathrm{A}_{2}$. 

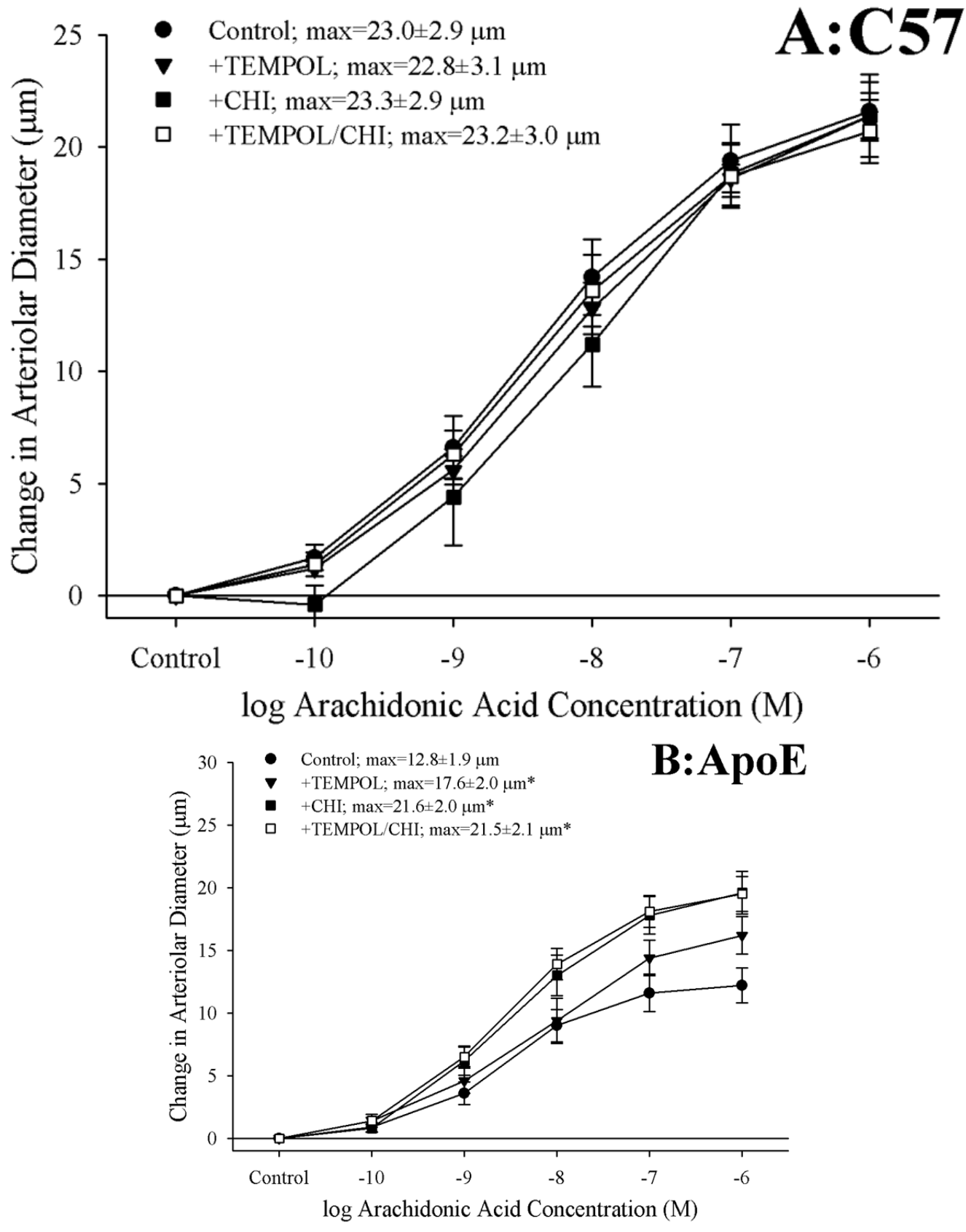


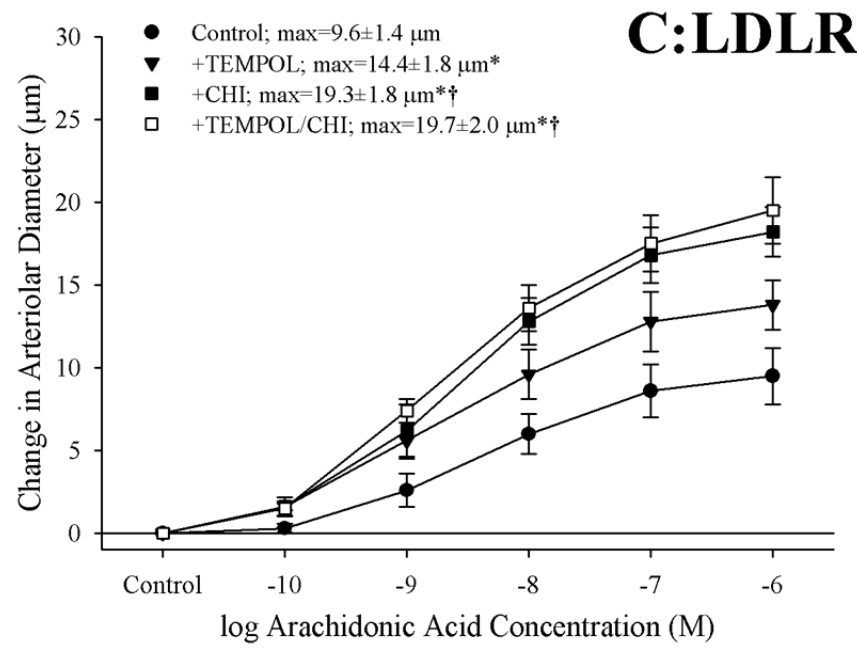

Figure 6.

Data, presented as mean \pm SEM, describing the dilator responses of isolated skeletal muscle resistance arterioles of C57 (Panel A), ApoE (Panel B) and LDLR (Panel C) mice in response to increasing concentrations of arachidonic acid. Data are shown for arterioles under control conditions, following treatment of vessels with the antioxidant TEMPOL, following pharmacological inhibition of thromboxane synthase with $\mathrm{CHI}$, and following treatment with both TEMPOL and CHI. $n=8-10$ animals for each group; $* \mathrm{p}<0.05$ vs. control conditions; $\dagger \mathrm{p}<0.05$ vs. treatment with TEMPOL alone. 


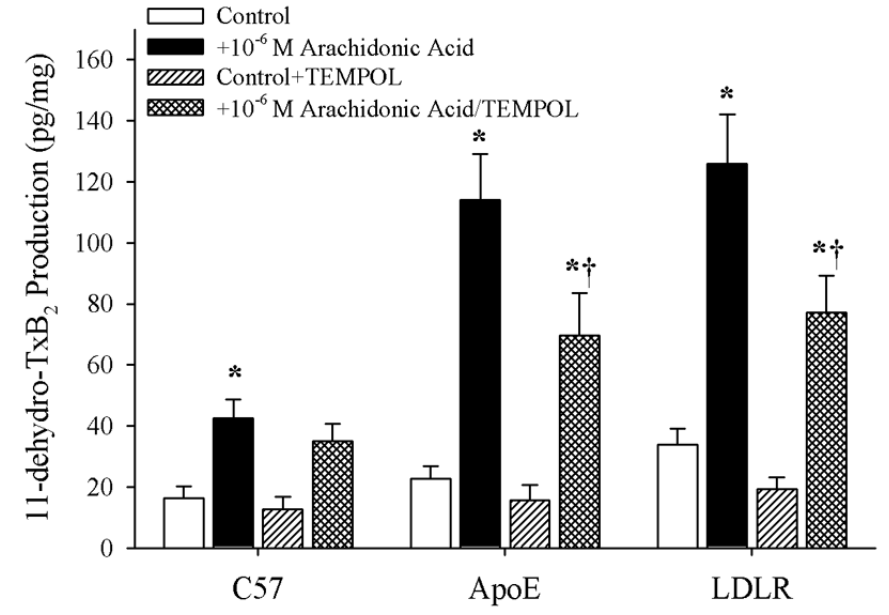

Figure 7.

Data describing the arterial production of thromboxane $\mathrm{A}_{2}$ (as 11-dehydro $\mathrm{TxB}_{2}$; Panel B) from C57, ApoE and LDLR in response to $10^{-6} \mathrm{M}$ arachidonic acid. Data, presented as mean \pm SEM, are shown under control conditions, and following treatment of pooled arteries with the antioxidant TEMPOL $\left(10^{-4} \mathrm{M}\right) . \mathrm{n}=6$ animals for each group, with each $\mathrm{n}$ representing pooled arteries from an individual mouse; please see text for details. $* \mathrm{p}<0.05$ vs. within-strain/no arachidonic acid; $\uparrow \mathrm{p}<0.05$ vs. within-strain/with arachidonic acid. 
Table 1

Baseline characteristics of mice and individual arterioles used in the present study

\begin{tabular}{|c|c|c|c|}
\hline & C57 & ApoE & LDLR \\
\hline Mass $(\mathrm{g})$ & $33 \pm 2$ & $34 \pm 2$ & $33 \pm 2$ \\
\hline MAP (mmHg) & $88 \pm 4$ & $92 \pm 3$ & $106 \pm 5^{* \dagger}$ \\
\hline$[\text { Glucose }]_{\text {blood }}(\mathrm{mg} / \mathrm{dl})$ & $84 \pm 7$ & $103 \pm 11$ & $115 \pm 7^{*}$ \\
\hline$[\text { Insulin }]_{\text {plasma }}(\mathrm{ng} / \mathrm{ml})$ & $1.1 \pm 0.3$ & $1.6 \pm 0.3$ & $2.8 \pm 0.5^{*}$ \\
\hline$[\text { Total Cholesterol }]_{\text {plasma }}(\mathrm{mg} / \mathrm{dl})$ & $88 \pm 9$ & $288 \pm 17^{*}$ & $364 \pm 22 * \dagger$ \\
\hline$[\text { LDL Cholesterol }]_{\text {plasma }}(\mathrm{mg} / \mathrm{dl})$ & $49 \pm 5$ & $260 \pm 11^{*}$ & $338 \pm 19^{* \dagger}$ \\
\hline$[\text { Triglycerides }]_{\text {plasma }}(\mathrm{mg} / \mathrm{dl})$ & $88 \pm 10$ & $175 \pm 14^{*}$ & $116 \pm 18^{\dagger}$ \\
\hline Nitrotyrosine $_{\text {plasma }}(\mathrm{ng} / \mathrm{ml})$ & $14 \pm 5$ & $54 \pm 11^{*}$ & $60 \pm 14^{*}$ \\
\hline Inner Diameter - Active $(\mu \mathrm{m})$ & $54 \pm 4$ & $55 \pm 5$ & $51 \pm 4$ \\
\hline Inner Diameter - Passive $(\mu \mathrm{m})$ & $128 \pm 5$ & $122 \pm 4$ & $118 \pm 7$ \\
\hline Active Tone (\%) & $57 \pm 3$ & $55 \pm 4$ & $56 \pm 4$ \\
\hline$<0.05$ vs. C57; & & & \\
\hline
\end{tabular}

